\title{
A few remarks on the relationship between elementary particle physics, gravitation and cosmology
}

\author{
V. M. Mostepanenko ${ }^{1}$ \\ ${ }^{1}$ Central Astronomical Observatory at Pulkovo of the Russian \\ Academy of Sciences, Saint Petersburg, 196140, Russia \\ and \\ Institute of Physics, Nanotechnology and Telecommunications, \\ Peter the Great Saint Petersburg Polytechnic University, Saint Petersburg, 195251, Russia \\ E-mail: vmostepa@gmail.com
}

\begin{abstract}
We discuss some current problems in the relationship between elementary particle physics and gravitation, i.e., in the subject investigated by Prof. K. P. Stanykovich half a century ago. Specifically, the inflationary stage of the Universe evolution, originating from the vacuum polarization and particle creation, corrections to Newton's gravitational law due to exchange of light elementary particles or spontaneous compactification of extra dimensions, and constraints on the parameters of axions as probable constituents of dark matter in our Universe are considered. It is pointed out that presently the relationship between elementary particles and gravitation has become an experimental science, and many experiments, directed towards resolution of all related problems, are performed in many countries.

PACS numbers: 14.80.Va, 12.20.Fv, 14.80.-j
\end{abstract}




\section{INTRODUCTION}

It is common knowledge that the main stream of physics in the middle of the twentieth century was very far from the areas studied by gravitation and cosmology. The major theoretical discoveries in fundamental physics at that time and later were the creation of quantum electrodynamics, unified theory of electroweak interaction and quantum chromodynamics. All these theories of the Standard Model were formulated in Minkowski space-time background and simply ignored any possible role of the gravitational interaction. In the second part of the twentieth century fundamental theoretical physics was dominated by the attempts of far-reaching generalizations of already constructed formalisms, based on the concepts of supersymmetry, supergravity and string theory, but they were not as fruitful as the Standard Model.

Professor K. P. Stanyukovich started his investigation of the relationship between the gravitational field and elementary particles at the time when almost everybody considered this subject as not prospective. There were no experimental results in the field. As a consequence, many admissions and conclusions made in the first book devoted to gravitational field and elementary particles [1], published by K. P. Stanyukovich, were of necessity largely hypothetical. His ideas met with considerable resistance on the source side of the official academic science. However, some of the results obtained in the book [1] found confirmation and were further developed by K. P. Stanyukovich together with his disciples and collaborators [2], and by other researches [3]. The point is that K. P. Stanyukovich was the great expert in the gas dynamics and, more generally, in the unsteady motion of continuous media, where his physical intuition allowed obtaining many universally recognized outstanding results. Just this intuition has probably helped him to work in the area of gravitational field and elementary particles, where solid theoretical framework was not yet developed and experimental data were still missing.

During the last half a century the situation in this field of science has been changed considerably. Although the quantum theory of gravitation is not yet available, the role of elementary particles in the evolution of the Universe is commonly recognized, and astrophysics and cosmology cannot obviate the need in particle physics. The quantum field theory in curved space-time allowed an understanding of the process of inflation at the very early stages of the Universe evolution. Particle physics suggests explanations for the ex- 
perimentally discovered acceleration of the Universe expansion, to the physical nature of dark matter, and to the great outputs of energy by some cosmic sources. A lot of experimental and observational data on the relationship between elementary particles, gravitation and cosmology has become available from both the laboratory and space experiments [4]. Presently this branch of physics is recognized as one of the most prospective.

In this paper, several selected topics on the relationship between elementary particle physics, gravitation and cosmology are addressed. In Sec. 2 we discuss the subject which goes back to the book [1], i.e., the particle creation and vacuum polarization at the very early stages of the evolution of the Friedmann Universe. Section 3 is devoted to the corrections to Newton's law of gravitation at short separation distances, which arise due to the effects of elementary particle physics. Possible role of light pseudoscalar particles, axions, as constituents of dark matter, is considered in Sec. 4. In Sec. 5 the reader will find our conclusions and a brief discussion.

Throughout the paper we use the system of units in which $\hbar=c=1$.

\section{QUANTUM FIELDS IN CURVED SPACE-TIME}

In the absence of workable quantum theory of gravitational field, the quantum field theory of matter fields in the gravitational background provides the reliable foundation for a solution of many important problems. The role of vacuum quantum effects of matter fields, such as vacuum polarization and particle creation, in the homogeneous isotropic gravitational background was investigated in the beginning of eightieths of the last century by solving the self-consistent Einstein equations

$$
G_{i k}+\Lambda g_{i k}=-8 \pi G \sum_{s}\left\langle 0\left|T_{i k}^{(s)}\right| 0\right\rangle_{\mathrm{ren}}
$$

Here, $G_{i k}$ and $g_{i k}$ are the Einstein and metrical tensors, $\Lambda$ and $G$ are the cosmological and gravitational constants, and the renormalized expectation values of the operator of stressenergy tensor of matter field of spin $s, T_{i k}^{(s)}$, are calculated in the vacuum state $|0\rangle$.

The explicit expressions for the vacuum expectation values of the stress-energy tensor of massless fields, entering Eq. (11), were obtained by a number of authors (see the monographs [3, 5]). Using these expressions, the self-consistent solutions of Eq. (1) with $\Lambda=0$ for the 
scale factor $a(t)$ of the space homogeneous isotropic metrics have been found [6]

$$
\begin{aligned}
& a(t)=\sqrt{\frac{G}{360 \pi}} \cosh \left(t \sqrt{\frac{360 \pi}{G}}\right), \\
& a(t)=\sqrt{\frac{G}{360 \pi}} \exp \left(t \sqrt{\frac{360 \pi}{G}}\right)
\end{aligned}
$$

in the cases of a spherical and flat 3 -space, respectively, where $t$ is the proper synchronous time and $s=0$. These solutions describe the de Sitter space-time with an exponentially increasing scale factor. Only a year later such scale factors have been obtained from the action of the so-called "inflaton" field and used in the theory of inflation [7]. It should be noted, however, that the classical inflaton field was introduced ad hoc, especially to obtain the solutions (2), whereas these solutions are directly obtainable by solving Eq. (1), i.e., from the first principles of quantum field theory in curved space-time and do not require any additional speculations.

Using the instabilities of the de Sitter solutions (2) relative to massive scalar modes, the inflationary cosmological scenario was constructed [8], where the exponentially fast expansion of the Universe, described by Eq. (2), transforms into the radiative dominated Friedmann expansion regime. An important role in this transformation is played by the effect of exponential increase of the number of scalar particles created from vacuum by the periodic in time external field [9] (see [10] for details). To date the inflationary scenario has received several observational confirmations in experiments intended to measure the anisotropy of cosmic microwave background radiation. Final confirmation could come from measurements of polarization of the background radiation induced by the relic gravitational waves, which were produced at the inflationary stage of evolution of the Universe. Such experiments are in operation. At the moment their results are, however, uncertain [11, 12] and no final conclusion is made yet. This demonstrates that presently the relationship between elementary particle physics, gravitation and cosmology has already become the experimental science. 


\section{PARTICLE PHYSICS AND CORRECTIONS TO NEWTON'S GRAVITA- TIONAL LAW}

One more subject of gravitational theory, where the elementary particle physics plays a decisive role, is the behavior of the gravitational force at short separation distances. As was shown long ago (see, e.g., monograph [13]) the exchange of light scalar particles predicted in many extensions of the Standard Model leads to the Yukawa-type corrections to Newton's law of gravitation. As a result, the gravitational potential between two pointlike particles with masses $m_{1}$ and $m_{2}$ located at the points $\boldsymbol{r}_{1}$ and $\boldsymbol{r}_{2}$ takes the form [13, 14]

$$
V\left(\left|\boldsymbol{r}_{1}-\boldsymbol{r}_{2}\right|\right)=-\frac{G m_{1} m_{2}}{\left|\boldsymbol{r}_{1}-\boldsymbol{r}_{2}\right|}\left(1+\alpha e^{-\left|\boldsymbol{r}_{1}-\boldsymbol{r}_{2}\right| / \lambda}\right) .
$$

Here, $\alpha$ is the interaction strength of the Yukawa-type addition to the standard, Newton's, gravitational potential and $\lambda=1 / M$ is its interaction range, where $M$ is the mass of a light particle. The corrections of Yukawa-type to Newton's law of gravitation are predicted also by the extra-dimensional theories of particle physics with a low-energy compactification scale [15, 16]. In this case the gravitational potential again has the form of (3), but the parameter $\lambda$ has the meaning of the characteristic size of a multidimensional compact manifold.

An exchange of one or even number of massless scalar particles leads to the power-type corrections to Newton's gravitational law with different powers

$$
V\left(\left|\boldsymbol{r}_{1}-\boldsymbol{r}_{2}\right|\right)=-\frac{G m_{1} m_{2}}{\left|\boldsymbol{r}_{1}-\boldsymbol{r}_{2}\right|}\left[1+\Lambda_{n}\left(\frac{r_{0}}{\left|\boldsymbol{r}_{1}-\boldsymbol{r}_{2}\right|}\right)^{n-1}\right],
$$

where $\Lambda_{n}$ is the interaction strength for different integer $n$, and $r_{0} \equiv 10^{-15} \mathrm{~m}$ is chosen to preserve the usual dimension of energy for different $n$. The potential (4) with $n=3$ is also predicted by the extra-dimensional theories with noncompact but warped extra dimensions [17, 18].

Nowadays, the investigation of relationship between the elementary particle physics and gravitation is an experimental science. Because of this, a lot of experiments has been performed in order to discover the corrections to Newton's law described by Eqs. (3) and (4) or at least to constrain their parameters. In the interaction region of $\lambda$ above a few micrometers, the strongest constraints on $\alpha$ follow from the gravitational experiments of Eötvos and Cavendish type (see [13, 19] for a review). It was shown [20, 21] that at shorter inter-

action ranges the most strong constraints are obtainable from the Casimir effect [22, 23]. 
In Fig. 1, we present the strongest constraints on $\alpha, \lambda$, which follow from the most precise experiments on measuring the Casimir interaction in the micrometer and submicrometer interaction ranges. The constraints, following from each experiment, are shown by one of the lines plotted in Fig. 1. In so doing, the regions of the $\alpha, \lambda$ plane above each line are prohibited by the measurement results and below each line are allowed.

Within the shortest interaction range from $\lambda=1.6$ to $11.6 \mathrm{~nm}$, the strongest constraints on $\alpha, \lambda$ shown by the line 1 were obtained [24] from measurements of the lateral Casimir force between sinusoidally corrugated surfaces [25, 26] (these constraints are stronger than the previously known ones [27] following from the experiment with two crossed cylinders). At larger $\lambda$ from 11.6 to $17.2 \mathrm{~nm}$ the strongest constraints of the line 2 were found [28] from measurements of the normal Casimir force between corrugated surfaces [29, 30]. The constraints of line 3 follow [31, 32] from indirect measurements of the Casimir pressure by means of micromachined oscillator. They are the strongest ones over the interaction range from 17.2 to $40 \mathrm{~nm}$. The line 4 was recently obtained from the so-called "Casimir-less" experiment, where the Casimir force was nullified by using the difference force measurements [33]. It presents the most strong constraints on $\alpha, \lambda$ within the wide interaction range from $40 \mathrm{~nm}$ to $8 \mu \mathrm{m}$. The line 5 shows the constraints obtained from the Cavendish-type

experiments [34, 35]. As is seen in Fig. 1, the strength of constraints increases with increasing $\lambda$, i.e., with decreasing mass $M$ of a scalar particle. At small $\lambda$ much work should be done in order to make the presently known constraints stronger.

The strongest constraints on the power-type corrections to Newton's gravitational law in Eq. (4) follow from the Eötvos- and Cavendish-type experiments. The list of these constraints for different values of $n$ can be found in [22, 36].

\section{AXIONS AND THE PROBLEM OF DARK MATTER}

Modern astrophysics and cosmology deal with problems which were not known in the middle of the twentieth century. One of the greatest is the problem of dark matter, i.e., the confirmed fact that approximately $80 \%$ of the matter in the Universe possessing the regular equation of state is invisible and its physical nature is unknown. The possible solution of this problem is again connected with elementary particle physics. The point is that some internal problems of the Standard Model (strong CP violation and resulting 
large electric dipole moment of a neutron allowed theoretically but experimentally excluded) caused an introduction of the Peccei-Quinn symmetry [37]. The spontaneous and dynamical breaking of this symmetry gives rise to a new uncharged pseudoscalar particle, an axion, which interacts very weakly with familiar particles of the Standard Model [38, 39]. As was found long ago (see, e.g., [40, 41]), axions can serve as the constituents of dark matter and, thus, provide the concurrent resolution for major problems of elementary particle physics, on the one hand, and astrophysics and cosmology, on the other hand. Only experiment can answer the question of whether the axions really exist in nature. Because of this, a lot of experiments for searching axions has been performed in different countries (see, e.g., [42] for a review) and even more are planned in near future.

In spite of numerous attempts to detect axions in both astrophysical and laboratory experiments, no solid evidence for their existence has been obtained yet. However, rather strong constraints on the parameters of axions (the mass and coupling constants to electrons, photons and nucleons) have been found. In so doing, the originally introduced axions were constrained to a very narrow region in the parameter space, and a lot of so-called axionlike particles were introduced. The point is that the original axions [37, 38] are the pseudo Nambu-Goldstone bosons and they are coupled to nucleons via the pseudovector Lagrangian density [40, 43]. As to the axion-like particles, introduced in different versions of the Grand Unified Theories, their coupling to fermions is described by the pseudoscalar Lagrangian density [40, 43].

The most reliable constraints on the coupling constants of axions and axion-like particles are obtained from different laboratory experiments. Here, we present several constraints on the axion-to-nucleon coupling constants found recently from measurements of the Casimir interaction between closely spaced test bodies [22, 23]. In this case, the additional force arising between two nucleons due to the two-axion exchange has been used. The problem is that the exchange of one axion between two nucleons leads to the spin-dependent interaction potential, which is averaging to zero in the case of nonpolarized test bodies used in experiments on measuring the Casimir interaction. The spin-independent interaction potential due to the exchange of two axions is known for only the pseudoscalar coupling of axions to nucleons and, thus, is applicable to only axion-like particles. It is given by [44]

$$
V\left(\left|\boldsymbol{r}_{1}-\boldsymbol{r}_{2}\right|\right)=-\frac{g_{a k}^{2} g_{a l}^{2}}{32 \pi^{3} m_{k} m_{l}} \frac{m_{a}}{\left(\boldsymbol{r}_{1}-\boldsymbol{r}_{2}\right)^{2}} K_{1}\left(2 m_{a}\left|\boldsymbol{r}_{1}-\boldsymbol{r}_{2}\right|\right) .
$$


Here, $g_{a k}$ and $g_{a l}$ are the axion-proton $(k, l=p)$ or axion-neutron $(k, l=n)$ coupling constants, $m_{k}$ and $m_{l}$ are the nucleon massres, $m_{a}$ is the axion mass, and $K_{1}(z)$ is the modified Bassel function of the second kind.

In Fig. 2 we present the constraints on the axion-to-nucleon coupling constants following from different experiments. Each line corresponds to one specific experiment. Similar to Fig. 1, the part of the plane $g_{a n(p)}, m_{a}$ above each line is excluded by the measurement data and below each line is allowed. The dashed lines 1 and 2 show the constraints obtained [45] from the Cavendish-type experiments [46, 47] and Eötvos-type experiment [48], respectively. The most strong gravitational constraints on the coupling constants of axion-like particles to nucleons shown by the line 3 were obtained [49] from the recent Cavendish-type experiment [50]. The dashed line 4 indicates the constraints found [51] from measurements of the thermal Casimir-Polder force between ${ }^{87} \mathrm{Rb}$ atoms of a Bose-Einstein condensate and an amorphous $\mathrm{SiO}_{2}$ plate [52] (see also the remark concerning the comparison of this experiment with theory [53]). The constraints on $g_{a n(p)}, m_{a}$ following [54] from measurements of the gradient of the Casimir force by means of an atomic force microscope [55] are shown by the dashed line 5. The line 6 shows the constraints obtained [56] from measurements of the effective Casimir pressure by means of micromachined oscillator [31, 32]. Measurements of the lateral Casimir force between sinusoidally corrugated surfaces [25, 26] lead to the constraints shown by the line 7 [57]. Finally, the line 8 demonstrates the constraints on $g_{a n(p)}, m_{a}$ following [58] from the recent Casimir-less experiment [33]. As can be seen in Fig. 2, the strength of constraints on $g_{a n(p)}$ increases with decreasing axion mass $m_{a}$. It is in close analogy with Fig. 1 demonstrating the constraints on the Yukawa-type corrections to Newton's law due to the exchange of one scalar particle.

\section{CONCLUSIONS AND DISCUSSION}

As is illustrated in the foregoing, there are relationships between problems of the elementary particle physics, on the one hand, and gravitation and cosmology, on the other hand. In this paper we have briefly discussed the three of them: vacuum polarization and particle creation, which determine the inflationary stage of the Universe evolution and its transition to the Friedmann stage; corrections to Newton's law of gravitation due to exchange of light elementary particles between atoms of macrobodies or due to spontaneous compactification 
of extra spatial dimensions; constraining the parameters of axions, as probable constituents of dark matter in the Universe. It is remarkable that all these, and many other problems typical for relationships between the elementary particle physics and gravitation, are presently of experimental character. Many experiments in different countries have been performed already for their resolution.

One can conclude that at the moment the role of elementary particle physics in gravitation, astrophysics and cosmology has gained wide recognition. Although first investigations of Prof. K. P. Stanyukovich in this field [1] were mostly of qualitative and hypothetical character, his ideas continue to attract a lot of interest worldwide. This is confirmed by the English translation of the book [59], where some of these ideas are presented in the popular form accessible for a general reader.

[1] K. P. Stanyukovich, Gravitational Field and Elementary Particles (Nauka, Moscow, 1965), in Russian.

[2] K. P. Stanyukovich and V. N. Melnikov, Gydrodynamics, Fields and Constants in Gravitational Theory (Energoatomizdat, Moscow, 1983), in Russian.

[3] A. A. Grib, S. G. Mamayev, and V. M. Mostepanenko, Quantum Effects in Intensive External Fields (Atomizdat, Moscow, 1980), in Russian; Vacuum Quantum Effects in Strong Fields (Friedmann Laboratory Publishing, St. Petersburg, 1994).

[4] M. Khlopov, Fundamentals of Cosmic Particle Physics (CISP-Springer, Cambridge, 2012).

[5] N. D. Birrell and P. C. W. Davies, Quantum Fields in Curved Space (Cambridge University Press, Cambridge, 1982).

[6] S. G. Mamayev and V. M. Mostepanenko, Sov. Phys. - JETP (USA) 51, 9 (1980) [Zh. Eksp. Teor. Fiz. 78, 20 (1980)].

[7] A. H. Guth, Phys. Rev. D 23, 347 (1981).

[8] A. A. Starobinsky, Phys. Lett. B 91, 99 (1980).

[9] V. M. Mostepanenko and V. M. Frolov, Sov. J. Nucl. Phys. 19, 451 (1974) [Yad. Fiz. 19, 885 (1974)].

[10] A. D. Linde, Particle Physics and Inflationary Cosmology (Harwood Academic, Chur, 1990).

[11] P. A. R. Ade et al. (BICEP2 Collaboration), Phys. Rev. Lett. 112, 241101 (2014). 
[12] W. Clavin, https://www.nasa.gov/jpl/gravitational-waves-from-early-universe-remain-illusive

[13] E. Fischbach and C. L. Talmadge, The Search for Non-Newtonian Gravity (Springer, New York, 1999).

[14] G. L. Klimchitskaya and V. M. Mostepanenko, Grav. Cosmol. 20, 3 (2014).

[15] I. Antoniadis, N. Arkani-Hamed, S. Dimopoulos, and G. Dvali, Phys. Lett. B 436, 257 (1998).

[16] N. Arkani-Hamed, S. Dimopoulos, and G. Dvali, Phys. Rev. D 59, 086004 (1999).

[17] L. Randall and R. Sundrum, Phys. Rev. Lett. 83, 3370 (1999).

[18] L. Randall and R. Sundrum, Phys. Rev. Lett. 83, 4690 (1999).

[19] E. G. Adelberger, J. H. Gundlach, B. R. Heckel, S. Hoedl, and S. Schlamminger, Progr. Part. Nucl. Phys. 62, 102 (2009).

[20] V. A. Kuzmin, V. A. Tkachev, and M. E. Shaposhnikov, JETP Lett. 36, 59 (1982) [Pis'ma v ZhETF 36, 49 (1982)].

[21] V. M. Mostepanenko and I. Yu. Sokolov, Phys. Lett. A 125, 405 (1987).

[22] M. Bordag, G. L. Klimchitskaya, U. Mohideen, and V. M. Mostepanenko, Advances in the Casimir Effect (Oxford University Press, Oxford, 2015).

[23] G. L. Klimchitskaya, U. Mohideen, and V. M. Mostepanenko, Rev. Mod. Phys. 81, 1827 (2009).

[24] V. B. Bezerra, G. L. Klimchitskaya, V. M. Mostepanenko, and C. Romero, Phys. Rev. D 81, $055003(2010)$.

[25] H.-C. Chiu, G. L. Klimchitskaya, V. N. Marachevsky, V. M. Mostepanenko, and U. Mohideen, Phys. Rev. B 80, 121402(R) (2009).

[26] H.-C. Chiu, G. L. Klimchitskaya, V. N. Marachevsky, V. M. Mostepanenko, and U. Mohideen, Phys. Rev. B 81, 115417 (2010).

[27] V. M. Mostepanenko and M. Novello, Phys. Rev. D 63, 115003 (2001).

[28] G. L. Klimchitskaya, U. Mohideen, and V. M. Mostepanenko, Phys. Rev. D 87, 125031 (2013).

[29] A. A. Banishev, J. Wagner, T. Emig, R. Zandi, and U. Mohideen, Phys. Rev. Lett. 110, $250403(2013)$.

[30] A. A. Banishev, J. Wagner, T. Emig, R. Zandi, and U. Mohideen, Phys. Rev. B 89, 235436 (2014).

[31] R. S. Decca, D. López, E. Fischbach, G. L. Klimchitskaya, D. E. Krause, and V. M. Mostepanenko, Phys. Rev. D 75, 077101 (2007). 
[32] R. S. Decca, D. López, E. Fischbach, G. L. Klimchitskaya, D. E. Krause, and V. M. Mostepanenko, Eur. Phys. J. C 51, 963 (2007).

[33] Y.-J. Chen, W. K. Tham, D. E. Krause, D. López, E. Fischbach, and R. S. Decca, arXiv:1410.7267v1.

[34] D. J. Kapner, T. S. Cook, E. G. Adelberger, J. H. Gundlach, B. R. Heckel, C. D. Hoyle, and H. E. Swanson, Phys. Rev. Lett. 98, 021101 (2007).

[35] A. A. Geraci, S. J. Smullin, D. M. Weld, J. Chiaverini, and A. Kapitulnik, Phys. Rev. D 78, $022002(2008)$.

[36] G. L. Klimchitskaya and V. M. Mostepanenko, Grav. Cosmol. 21, 1 (2015).

[37] R. D. Peccei and H. R. Quinn, Phys. Rev. Lett. 38, 1440 (1977).

[38] S. Weinberg, Phys. Rev. Lett. 40, 223 (1978).

[39] F. Wilczek, Phys. Rev. Lett. 40, 279 (1978).

[40] J. E. Kim, Phys. Rep. 150, 1 (1987).

[41] Yu. N. Gnedin, Int. J. Mod. Phys. A 17, 4251 (2002).

[42] K. Baker et al. Ann. Phys. (Berlin) 525, A93 (2013).

[43] G. G. Raffelt, J. Phys. A: Math. Theor. 40, 6607 (2007).

[44] S. D. Drell and K. Huang, Phys. Rev. 91, 1527 (1953).

[45] E. G. Adelberger, E. Fischbach, D. E. Krause, and R. D. Newman, Phys. Rev. D 68, 062002 (2003).

[46] R. Spero, J. K. Hoskins, R. Newman, J. Pellam, and J. Schultz, Phys. Rev. Lett. 44, 1645 (1980).

[47] J. K. Hoskins, R. D. Newman, R. Spero, and J. Schultz, Phys. Rev. D 32, 3084 (1985).

[48] G. L. Smith, C. D. Hoyle, J. H. Gundlach, E. G. Adelberger, B. R. Heckel, and H. E. Swanson, Phys. Rev. D 61, 022001 (1999).

[49] E. G. Adelberger, B. R. Heckel, S. Hoedl, C. D. Hoyle, D. J. Kapner, and A. Upadhye, Phys. Rev. Lett. 98, 131104 (2007).

[50] D. J. Kapner, T. S. Cook, E. G. Adelberger, J. H. Gundlach, B. R. Heckel, C. D. Hoyle, and H. E. Swanson, Phys. Rev. Lett. 98, 021101 (2007).

[51] V. B. Bezerra, G. L. Klimchitskaya, V. M. Mostepanenko, and C. Romero, Phys. Rev. D 89, 035010 (2014).

[52] J. M. Obrecht, R. J. Wild, M. Antezza, L. P. Pitaevskii, S. Stringari, and E. A. Cornell, Phys. 
Rev. Lett. 98, 063201 (2007).

[53] G. L. Klimchitskaya and V. M. Mostepanenko, J. Phys. A: Math. Theor. 41, 312002 (2008).

[54] V. B. Bezerra, G. L. Klimchitskaya, V. M. Mostepanenko, and C. Romero, Phys. Rev. D 89, $075002(2014)$.

[55] C.-C. Chang, A. A. Banishev, R. Castillo-Garza, G. L. Klimchitskaya, V. M. Mostepanenko, and U. Mohideen, Phys. Rev. B 85, 165443 (2012).

[56] V. B. Bezerra, G. L. Klimchitskaya, V. M. Mostepanenko, and C. Romero, Eur. Phys. J. C 74, 2859 (2014).

[57] V. B. Bezerra, G. L. Klimchitskaya, V. M. Mostepanenko, and C. Romero, Phys. Rev. D 90, 055013 (2014).

[58] G. L. Klimchitskaya and V. M. Mostepanenko, Eur. Phys. J. C 75, 164 (2015).

[59] M. Vasilyev and K. P. Stanyukovich, Matter and Man (University Press of the Pacific, Honolulu, 2000). 


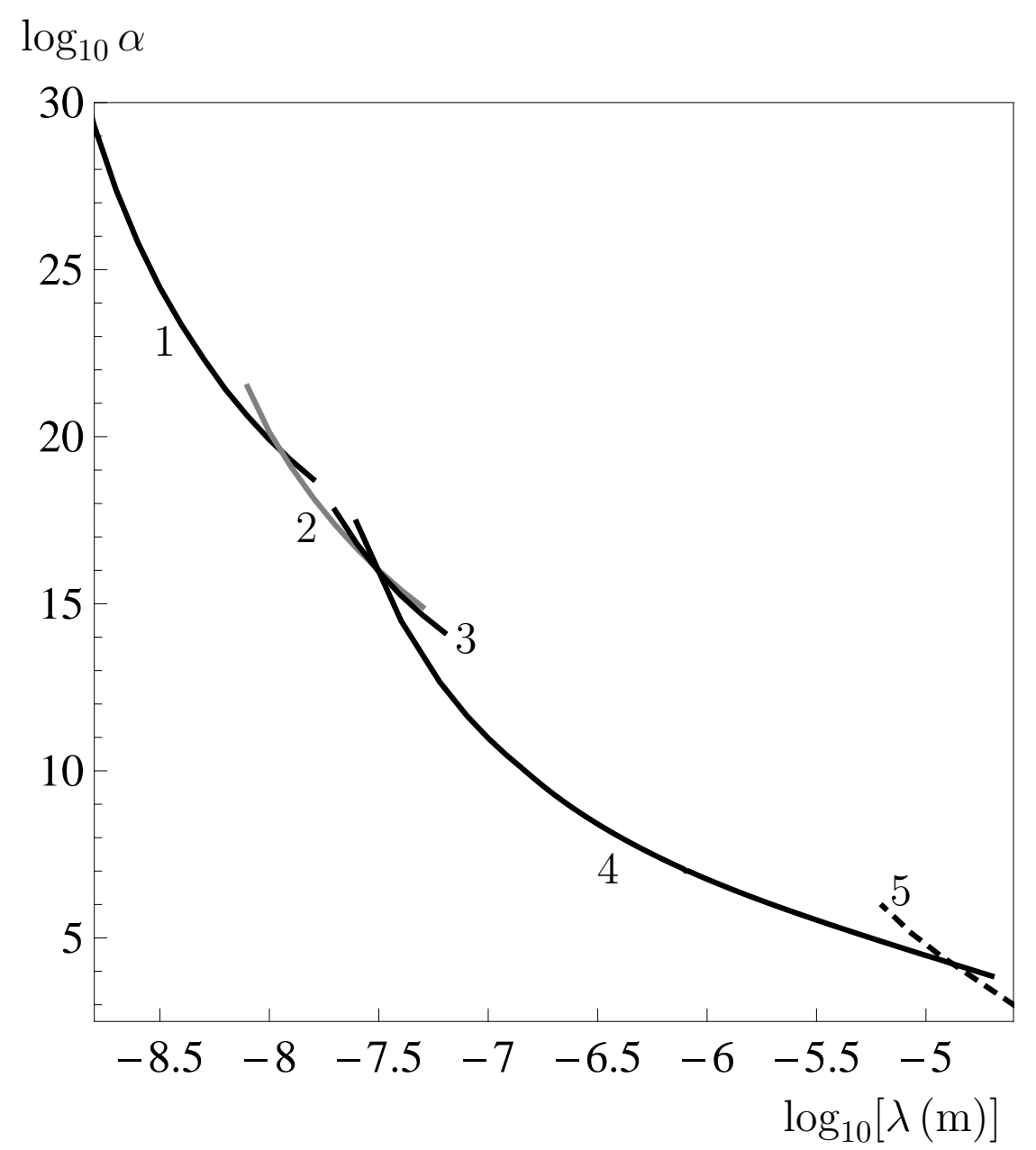

FIG. 1: Strongest constraints on the Yukawa-type corrections to Newton's law of gravitation obtained from different measurements of the Casimir force (the lines 1-4) and from Cavendishtype experiments (the line 5). The regions above each line are prohibited and below each line are allowed. See text for further discussion. 


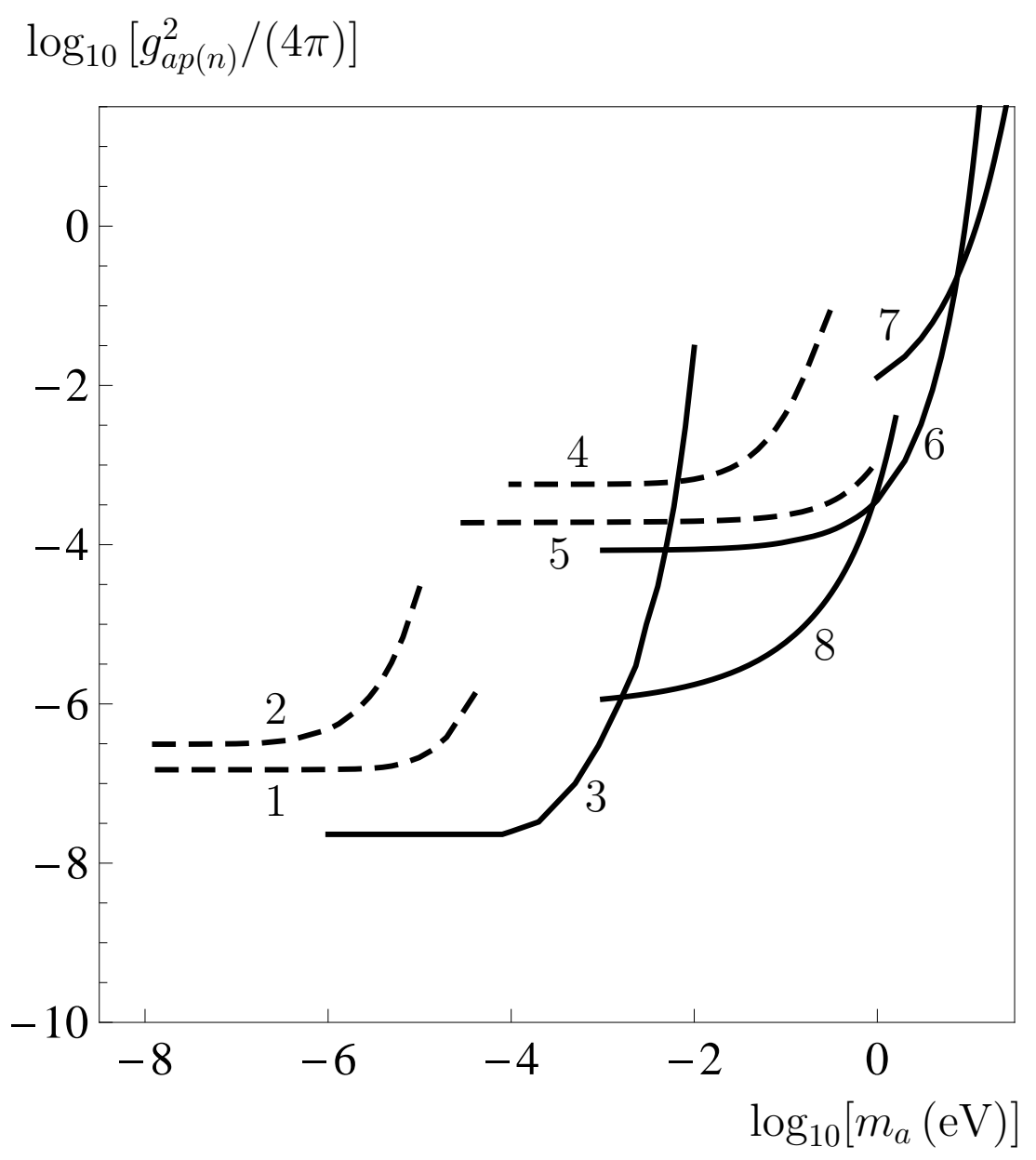

FIG. 2: Constraints on the axion-to-nucleon coupling constants following from the Cavendish-type (the lines 1 and 3) and Eötvos-type (the line 2) experiments and from measurements of the CasimirPolder and Casimir interactions (the lines 4-8). The regions above each line are prohibited and below each line are allowed. See text for further discussion. 